\title{
Gedanken zu dem Nomen זרע, „Samen, Nachkommen" in Genesis
}

\author{
WILFRIED WARNING (SCHULZENTRUM MARIENHÖHE, DARMSTADT)
}

\begin{abstract}
In some recent studies that scrutinized selected passages of the Hebrew Bible, the existence of linguistic linkages has been shown. Evidently, the so-called "terminological patterns" are one of the structural devices by means of which the extant Endgestalt, the final form, has been crafted. In Genesis the noun זרע "seed, offspring," appears fifty-nine times, both in MT, the Samaritan Pentateuch and LXX's Hebrew source text. The fifty-nine occurrences are part of "priestly" and "non-priestly" texts. The three terminological patterns coming to light are intricately intertwined and emphasize three special promises (of descendants). In the 59-part table (Gen 1:150:26) the metaphor "your descendants ... like the dust of the earth" takes the fifteenth position (13:16a) and the fifteenth-from-last (28:14a). In the 47-part equidistant structure (Gen 11:27-50:26) the metaphor "your descendants ... as the stars of heaven" shows in the twenty-first text (22:17a) and the twenty-first-from-last (26,4a). In the 25-part table (11:27-25:11) the phrase "I will establish my covenant ... and your/his descendants" holds the tenth position (17:7a) and the tenth-from-last (17:19b).
\end{abstract}

KEYWORDS: זרע; seed; offspring; priestly texts; Genesis.

\section{A GENESIS 1,1-50,26}

In seiner Studie Reading the Fractures of Genesis mahnt D. M. Carr, „careful synchronic study of a text's present form is an essential prelude to methodologically controlled reflection on how that form was produced." Der vorliegende, synchron ausgerichtete Aufsatz soll die pointierte Placierung des Wortes „Samen“ im jetzigen Genesistext näher beleuchten, denn close reading, gepaart mit intensiver Konkordanzarbeit, zeitigt die strukturierende Funktion des Wortes in der Genesis-,,Letztfassung." ${ }^{2}$ Das tradierte Textgefüge stellt (an diesem Punkt) ein stringent konzipiertes Ganzes dar. ${ }^{3}$

* Article submitted: 10/05/2017; peer-reviewed: 28/06/2017; accepted: 24/07/2017. Wilfried Warning, "Gedanken zu dem Nomen זרע ,Samen, Nachkommen” in Genesis," OTE no. 3 (2017): 852-873. DOI: https://doi.org/10.17159/2312-3621/2017/v30n3a18

1 David M. Carr, Reading the Fractures of Genesis (Louisville: Westminster John Knox Press, 1996), viii.

2 Georg Fischer, „Zur Lage der Pentateuchforschung,” ZAW 115 (2003): 615.

3 Thomas Hieke, Die Genealogien der Genesis, HBSt 39 (Freiburg: Herder, 2003), 
Im ersten Buch der Bibel kommt das Nomen neunundfünfzig Mal vor, zwölf Mal in der Urgeschichte ${ }^{4}(1,112.122 .292 ; 3,152 ; 4,25 ; 7,3 ; 8,22 ; 9,9)$, sechsunddreißig Mal in den Erzelternerzählungen (12,7; 13,15.162; 15,3.5.13.18; 16,10; 17,72.8.9.10. 12.19; 19,32.34; 21,12.13; 22,172.18; $24,7.60 ; 26,3.43 .24 ; 28,4.13 .142 ; 32,13 ; 35,12)$ und elfmal in der Josephgeschichte $^{5}(38,8.92 ; 46,6.7 ; 47,19.23 .24 ; 48,4.11 .19)$. Sein Bedeutungsspektrum reicht von samentragenden Pflanzen (1,112.122.292), dem Saatkorn (47,19.23.24) und dem jahreszeitlichen Wortpaar זרע וקציר ,Saat und Ernte“ $(8,22)$ bis hin zu den Tieren in der Arche $(7,3)$, „damit auf der ganzen Erde Samen am Leben erhalten bleibt." Gen 3,152 zielt ab auf den Samen der Frau bzw. der Schlange. Gen 4,25 hat Set im Blick (,denn Gott hat mir einen anderen Samen gegeben“), 9,9 die Nachfahren der Söhne Noahs (,ich richte auf meinen Bund mit euch und eurem Samen nach euch") und 19,32.34 die (Kindes)Kinder, welche Lots Töchter sich durch den Beischlaf mit ihrem Vater erhoffen. Die übrigen Texte richten unser Augenmerk auf die Nachkommen der Erzeltern: 12,$7 ; 13,15.162 ; 15,3.5 .13 .18 ; 16,10 ; 17,72.8 .9 .10$. 12.19; 21,12.13; 22,172.18; $24,7.60 ; 26,3.43 .24 ; 28,4.13 .142 ; 32,13 ; 35,12 ; 38,8.92 ; 46,6.7 ; 48,4.11 .19$.

LXX gibt das Nomen stets mit $\sigma \pi \varepsilon ́ p \mu a$ wieder. Beim Bundesschluss zwischen Abimelech und Abraham (Gen 21,22ff) fordert der König von Gerar $(20,2)$ den Erzvater auf, ,darum schwöre mir hier bei Gott, dass du mich, meinen

bemerkt: „Die Lektüre der Genesis von den Genealogien her lässt das erste Buch der Bibel als ein schlüssig gestaltetes und stringent ausgebautes literarisches Werk erscheinen, das im Zusammenhang lesbar ist."

4 Hieke, Genealogien, 250-251, konstatiert, „dass die weit verbreitete Unterscheidung zwischen einer ,Urgeschichte' und einer ,Vätergeschichte' der Gesamtstruktur des Buches Genesis nicht gerecht wird ... Der vorliegende Text unterscheidet nicht in ,Urgeschichte' und ,Vätergeschichte,' sondern erzählt in fortschreitender Fokussierung die Entstehung Israels inmitten der Menschheit.“

5 Der Entstehungsprozess der Josephgeschichte liegt verborgen im Dunkel der Literargeschichte Israels, so dass wir nicht wissen, wer sie wann verfasst und wer sie wann an ihren kanonischen Ort gestellt hat. Für Konrad Schmid, „Die Josephsgeschichte im Pentateuch," in Abschied vom Jahwisten: Die Komposition des Hexateuch in der jüngsten Diskussion, hg. Jan C. Gertz, Konrad Schmid, und Markus Witte, BZAW 315 (Berlin: de Gruyter, 2002), 94, ,ist sie quellenhaft und nicht von vornherein kontextgebunden entstanden.“ Albert de Pury, „Gottesname, Gottesbezeichnung und Gottesbegriff: 'Elohim als Indiz zur Entstehungsgeschichte des Pentateuch," in Abschied vom Jahwisten: Die Komposition des Hexateuch in der jüngsten Diskussion, Hg. Jan C. Gertz, Konrad Schmid, und Markus Witte, BZAW 315 (Berlin: de Gruyter, 2002), 46, postuliert: „P kennt noch keine Josephgeschichte, und diese gehört aus ganz anderen Gründen zur nachpriesterlichen Zeit.“ Thomas Römer, „Zwischen Urkunden, Fragmenten und Ergänzungen: Zum Stand der Pentateuchforschung," ZAW 125 (2013): 14, vermutet, dass die „Herkunft der Josephsnovelle ... aus der perserzeitlichen oder früh-hellenistischen jüdischen Diaspora zumindest eine bedenkenswerte Hypothese" ist. 
Sproß נין) und meinen Schoß (נכד) na nicht betrügen wirst. "'7 In 21,23 überträgt LXX das Wort נין ebenfalls mit $\sigma \pi \varepsilon \dot{p} p \mu \alpha$, so dass die 59-teilige Tabelle nur bedingt $\mathrm{zu}$ erkennen ist. Zu beachten ist indes, das Nomen ist in MT, dem Samaritanischen Pentateuch ${ }^{8}$ und (der hebräischen Vorlage der) LXX neunundfünfzig Mal bezeugt - eine gut gesicherte Ausgangsbasis.

Werden möglichst alle Wörter einer in sich geschlossenen Texteinheit, sei es ein kurzer Abschnitt, ein Kapitel oder wie in diesem Aufsatz ein biblisches Buch tabellarisiert, erfassen wir die relative Häufung und strukturale Position einzelner Wörter bzw. Wendungen. Hierbei treten drei Arten verbaler Verknüpfungen zutage, die „Inklusio,“9 die „offene Inklusio ${ }^{10 “}$ und die ,äquidistante Struktur."11 Sie fußen auf drei Kriterien, erstens, sie sind terminologisch und nicht nur inhaltlich ausgerichtet; zweitens, sie beruhen auf dem charakteristischen Gestaltungsmittel der Wiederaufnahme ${ }^{12}$; drittens, das entscheidende Moment ist jedoch die ,arithmo-logische“ Balance, die jede derartige Querverstrebung aufweist. Bei einem derartigen „Trial-and-Error" Prozess hat sich dieser Aspekt als probates Korrektiv erwiesen, denn die äquidistante Setzung des wieder aufgenommenen Textelements unterscheidet verbale Verknüpfungen eindeutig von Querbezügen, die einander zwar inhaltlich und terminologisch entsprechen, jedoch nicht ,,arithmo-logisch“ ausgerichtet sind. Ob der komplexen und vielschichtigen terminologischen Verknüpfungen stellt sich die Frage: Wer hat im Laufe der Literargeschichte des Buches Genesis ungleiche

6 Der hebräischen Alliteration נין ונכד nachspürend übersetzt Benno Jacob, Das Buch Genesis (Stuttgart: Calwer Verlag, 2000), 486, die Nomina mit „Sproß und „Schoß.“

7 Das dreimal im Tanach bezeugte Wortpaar kommt stets zusammen vor: Gen 21,23; Js 14,22; Hi 18,19. LXX überträgt es als $\sigma \pi \varepsilon \dot{\rho} \mu \alpha$ und övora.

8 Vgl. Abraham Tal und Moshe Florentin, Hg., The Pentateuch: The Samaritan Version and the Masoretic Version, Hebräisch (Tel Aviv: The Haim Rubin Tel Aviv University Press, 2010).

9 Vgl. Wilfried Warning, Literary Artistry in Leviticus, BibInt 35 (Brill: Leiden, 1999), 159-160; Wilfried Warning, ,Terminological Patterns and Genesis 24,” EstBíb 60 (2002): 62-63; Wilfried Warning, „Terminological Patterns and the Book of Esther," OTE 15 (2002): 491-496.

10 Vgl. Wilfried Warning, „Terminologische Verknüpfungen und Genesis 15, "Hen 23 (2001): 412-414; Warning, Leviticus, 115-120, 160-163; Wilfried Warning, „Terminological Patterns and Genesis 17,” HUCA 70/71 (2002): 102-103; Warning, „Esther,” 496-497, 501-502.

11 Vgl. Wilfried Warning, „Terminological Patterns and the Decalogue,” ZAW 118 (2006): 7-9; Warning, „Genesis 17,” 97-98; Warning, „Esther,” 497-499.

12 Vgl. Erhard Blum, Die Komposition der Vätergeschichte, WMANT 57 (Neukirchen-Vluyn: Neukirchener Verlag, 1984), 9; Moshe Anbar, „La 'Reprise',” VT 38 (1988), differenziert zwischen ,reprise secondaires” (386-393) und „reprises originales“ (393-398). Wird der Kontext durch die Wiederaufnahme unterbrochen, liegt s.E. eine sekundäre Wiederaufnahme vor, falls nicht, handelt es sich um eine originäre Wiederaufnahme. 
Warning, “Gedanken zu dem Nomen,” OTE 30/3 (2017): 852-873

Texte so miteinander verflochten, dass die pointierte Placierung bestimmter Aussagen an diversen Stellen aufblitzt? Diese Art Struktur schaffender Querverweise findet sich in kürzeren Texten, ${ }^{13}$ in Großabschnitten wie Genesis ${ }^{14}$ und sogar im Pentateuch. ${ }^{15}$

Nach Maßgabe des „Leitwortstils“ ist als Leitwort „ein Wort oder ein Wortstamm zu verstehen, der sich innerhalb eines Textes, einer Textfolge, eines Textzusammenhangs sinnreich wiederholt.“"16 Die „maßhafte Wiederholung“" eines Wortes oder Wortstammes ist nach M. Buber „wohl überhaupt das stärkste unter allen Mitteln, einen Sinncharakter kundzutun, ohne ihn vorzutragen. ${ }^{\text {"17 }}$ Die 59-teilige Tabelle beruht nicht auf dem Wortstamm זרע, sondern auf dem Substantiv. Dagegen basiert die offene Inklusio, welche die beiden Dekalogversionen in Ex 20 und Dtn 5 miteinander verzahnt, auf dem Wortstamm פל „(be)hauen; (aus Stein) gehauenes Gottesbild."18 Auch hier entscheidet ein „Trial-and-Error“ Ansatz darüber, ob eine verbale Verknüpfung auf dem Wortstamm oder dem Nomen / Verb beruht. Daher sind die sechs Belege des Zeitwortes „säen“ (Gen 1,11.12.292; 26,12; 47,23) nicht in die 59-teilige äquidistante Struktur eingebunden.

In seiner Abhandlung „Die Wiederholung als literarisches Phänomen“19 bezeichnet D. Dieckmann Wiederholungen, die er in seiner Studie zu Gen 26 aufzeigt, als „narrativ-repetitive[] Strukturen “20 und resümiert:

Dieser Durchgang durch verschiedene Konzepte für den Umgang mit dem literarischen Phänomen der Wiederholung hat gezeigt, dass die Interpretationen von Repetition und Variation so unterschiedlich sind wie die hermeneutischen Voraussetzungen der Lektüreweisen, in die sie eingebettet sind. Wie ich Wiederholungen wahrnehme, hängt davon $\mathrm{ab}$, wie ich lese. ${ }^{21}$

In der 59-teiligen Tabelle, sie umschließt nahezu das gesamte Buch Genesis, kommt die Metapher „dein Samen ... wie der Staub der Erde“ zweimal vor

13 Vgl. Wilfried Warning, ,Terminological Patterns and Genesis 39, 'JETS 44 (2001): 412-413; Warning, „Genesis 15,” 4-6; Warning, „Genesis 17,” 97-100; Warning, „Genesis 24,” 56-69; Warning, „Decalogue,” 514-515.

14 Vgl. Warning, „Genesis 15,” 6-7; Warning, „Genesis 17,” 105-106; Warning, „Genesis 24,” 69-74.

15 Vgl. Warning, „Genesis 15,” 7-9; Warning, „Decalogue,” 518-521.

16 Martin Buber, „Leitwortstil in den Erzählungen des Pentateuch,“ in Schriften zur Bibel 2, (München: Kösel Verlag, 1964), 1131.

17 Buber, „Leitwortstil,“ 1131.

18 Warning, „Decalogue,“ 520-522.

19 Detlef Dieckmann, Segen für Isaak: Eine rezeptionsästhetische Auslegung von Genesis 26 und Kotexten, BZAW 329 (Berlin: de Gruyter, 2003), 11-105.

20 Dieckmann, Isaak, 103.

21 Dieckmann, Isaak, 103. 
$(13,16 a ; 28,14 a)$. Wie wir die Wiederaufnahme von 13,16 a in 28,14 a deuten, hängt also auch von den hermeneutischen Voraussetzungen $a b$, die uns beim Lesen leiten. Die an Abraham gerichtete Zusage in 13,16a entspricht der Verheißung, die Jakob nach 28,14a erhält, dies ist allseits bekannt. Unbekannt ist bisher die ,arithmo-logische“ Balance, die im tradierten Textgefüge aufleuchtet - vor Gen 13,16a ist das Nomen „Samen“ vierzehn Mal bezeugt und vierzehn Mal nach 28,14a. Wenn die Erzelternerzählungen und die Josephgeschichte als „literarische Einheit" betrachtet werden, zeigt sich eine 47-teilige Tabelle, in der die Metapher „dein Samen ... wie die Sterne des Himmels“ im einundzwanzigsten Text vorliegt $(22,17 \mathrm{a})$ und im einundzwanzigletzten $(26,4 \mathrm{a} \alpha){ }^{22}$ Im Abrahamzyklus ist das Nomen fünfundzwanzig Mal belegt. Diese äquidistante Struktur zeichnet sich dadurch aus, dass der im Tanach nur zweimal bezeugte Ausdruck ,einen Bund aufrichten ... mit deinem / seinem Samen ... als ewigen Bund“ (Gen 17,7a.19b) im zehnten Text (17,7a) und im zehnletzten $(17,19 \mathrm{~b})^{23}$ vorliegt. Könnte Sollte all das einer (nach)endredaktionellen Zufälligkeit geschuldet sein? Angesichts der Komplexität und Vielschichtigkeit der drei verbalen Verknüpfungen ist die Frage m. E. zu verneinen.

In der Diskussion um den Formulierungsprozess des Pentateuch misst die kritische Forschung dem „nicht-priesterlichen“24 (np) Gen 15 und dem

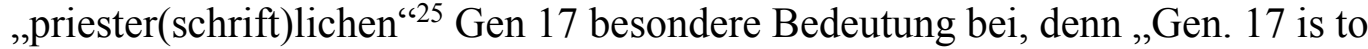
the Abraham cycle," so J. Ha, ,what Gen. 15 is to the entire Pentateuch. " ${ }^{26}$ Für

$22 \mathrm{Ob}$ und inwieweit die Erzelternerzählungen und die Josephgeschichte miteinander verflochten sind, wird kontrovers diskutiert - ein Konsens zeichnet sich nicht ab. Die subtile Setzung des Eigennamen ראובן,Ruben“ (29,32; 30,14; 35,22.23; 37,21.22.29; 42,22.37; 46,8.9; 48,5; 49,3) zeitigt eine gelungene Verflechtung von Erzelternerzählungen und Josephgeschichte: In der 13-teiligen Tabelle, die in MT, ראובן Samaritanus und LXX vorliegt, hat der im Tanach nur zweimal belegte Ausdruck Ruben, der Erstgeborene Jakobs“ den vierten $(35,23)$ und viertletzten $(48,8)$ Platz inne. Auch entspricht der sechste Text (37,22: „Ruben sagte zu ihnen: ,Vergießt kein Blut! Werft ihn in diese Zisterne ... aber legt nicht Hand an ihn'“) dem sechsletzten $(42,22$ : „Hatte ich euch nicht gesagt: ,Versündigt euch nicht an dem Jungen, aber ihr habt nicht gehört, und nun wird von uns Rechenschaft für sein Blut gefordert'“).

23 Vgl. Warning, „Genesis 17,” 97-98.

24 Vgl John Ha, Genesis 15: A Theological Compendium of Pentateuchal History, BZAW 181 (Berlin: de Gruyter, 1989), 216: „Given the equally dominant Deuteronomistic, Priestly as well as prophetic traits in Gen. 15, it is impossible to identify its author with any of these schools ... It may well be possible that he did not belong to any of these schools but was just an official scribe or teacher who saw the need to compose his work."

25 Vgl. Sean E. McEvenue, The Narrative Style of the Priestly Writer, AnBib 50 (Rom: Biblical Institute Press, 1971), 159: „In Gen 17, the priestly writer has combined materials from various pentateuchal sources, and other unknown sources."

26 Ha, Genesis 15, 205. Vgl. Benjamin Ziemer, Abram - Abraham: Kompositionsgeschichtliche Untersuchungen zu Genesis 14, 15 und 17, BZAW 350 (Berlin: de 
Warning, “Gedanken zu dem Nomen,” OTE 30/3 (2017): 852-873

B. Ziemer stammen sie ,angesichts deutlicher stilistischer und theologischer Differenzen kaum von demselben Verfasser, es ist aber kaum anzunehmen, dass sie völlig unabhängig voneinander entstanden sind.“27 Da eine gewisse Interdependenz nicht zu leugnen ist, stellt Ha die Frage:

Genesis 17 is another chapter loaded with promises. In fact most of the promises it contains are similar to those in Gen. 15: posterity, land and son. An obvious question that arises is: is there no redundancy between the two chapters? If none, what is the position of one in respect of the other? ${ }^{28}$

Neben den Themen Nachkommen, Land und Sohn wissen beide Kapitel vom Bundesschluss zwischen Gott und Abraham. Anhand der „Sinaireminiszenzen“ in Gen 15 ist, so J. C. Gertz, in Relation zu Gen 17 festzuhalten, dass ,die Abraham gewährte Offenbarung keine geringere gewesen ist als diejenige, die

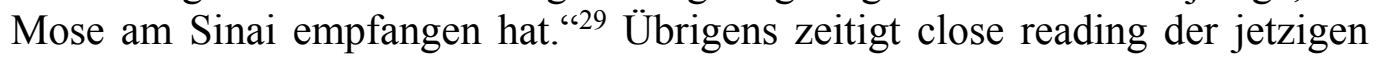
Abrahamgeschichte verbal fundierte und ,arithmo-logisch“ ausgerichtete Querverstrebungen, welche die zwei Kapitel miteinander verquicken.

Gruyter, 2005), 388-389; Erhard Blum, „Die literarische Verbindung von Erzvätern und Exodus: Ein Gespräch mit neueren Endredaktionshypothesen," in Abschied vom Jahwisten: Die Komposition des Hexateuch in der jüngsten Diskussion, hg. Jan C. Jan C. Gertz, Konrad Schmid, und Markus Witte, BZAW 315 (Berlin: de Gruyter, 2002), 141.

27 Benjamin Ziemer, „Die aktuelle Diskussion zur Redaktionsgeschichte des Pentateuch und die empirische Evidenz nach Qumran," ZAW 125 (2013): 384.

28 Ha, Genesis 15, 205.

29 Jan C. Gertz, „Abraham, Mose und der Exodus: Beobachtungen zur Redaktionsgeschichte von Gen 15," in Abschied vom Jahwisten: Die Komposition des Hexateuch in der jüngsten Diskussion, hg. Jan C. Gertz, Konrad Schmid, und Markus Witte, BZAW 315 (Berlin: de Gruyter, 2002), 77-78; vgl. Blum, „Verbindung,” 142: „Im Falle von Gen 15 hat sich in jüngster Zeit ein gewisser Forschungstrend herausgebildet, wonach dieses Kapitel bereits die priesterliche Überlieferung mit der Abraham- $b^{e}$ rît in Gen 17 voraussetzt.“ Ziemer, Abram - Abraham, 180, insistiert dagegen, dass keines „der in der Literatur angeführten Argumente ... die Abhängigkeit eines Versteiles, eines Verses, eines Abschnitts oder des ganzen Kapitels Gen 15 von Texten der Gen17-Schicht beweisen“ kann. Es lasse sich vielmehr zeigen, dass „Gen 15 in Gen 17 sowohl vorausgesetzt als auch verarbeitet wird“ (171). Mit Blick auf derart gegensätzliche Thesen konstatiert Fischer in „Pentateuchforschung,” 608: In der Forschung unterscheiden sich ,die vorgetragenen Positionen oft so sehr, daß sie kaum miteinander zu vereinbaren sind und sich die Frage stellt, was denn richtig sei.“ 
Ist Gen $15^{30}$ „strukturell einheitlich, und dennoch ,vorpriesterlich"“31? Gehört es ,zumindest in seiner ,kanonischen' Form zu den jüngsten Genesistexten" ${ }^{\text {"32? }}$ ? Inwieweit untermauert die tradierte Textgestalt Ziemers These, Gen 17 sei das ,theologische[] Schlüsselkapitel der Genesis“33? Wann und von wem sind Gen 15 und Gen 17 per se verfasst und von wem sind sie wann an ihren kanonischen Ort placiert worden? Diese Fragen sind nur hypothetisch zu beantworten, denn über den Formulierungsprozess des jetzigen Genesistextes liegt uns keine konkrete Information vor. Die deutlich divergierenden und sich teilweise ausschließenden Theoriemodelle, die in der derzeitigen Pentateuchforschung diskutiert werden, indizieren, dass bei genauerem Hinschauen sich jede These als eine mehr oder minder gute Hypothese erweist. Gemeinhin gilt die Prämisse, der vorliegende Genesistext sei weder in einem einzigen Formulierungsprozess entstanden noch zeichne er sich als ein Werk aus, das von Anfang bis Ende auf Kohärenz hin redigiert worden ist. ${ }^{34}$ Zudem ist man der Ansicht, das uns überkommene Textgefüge der Genesis sei das Resultat eines äußerst komplexen und langwierigen Entwicklungsprozesses. ${ }^{35}$ Nach R. G. Kratz ist „Das sicherste Ergebnis der Pentateuchkritik der vergangenen 200 Jahre, wenn man so will ihr ,archimedischer Punkt,' ... die Isolierung der Priesterschrift (P).“"36 Jede hier

30 Ziemer, Abram - Abraham, 166: „Der Text gehört zu jenen Textzusammenhängen, welche dem Verfasser der Endkomposition des Buches Genesis als des ersten Buches der Tora bereits in fester Zusammenstellung vorgelegen haben."

31 Ziemer, Abram - Abraham, 173; Ha, Genesis 15, 215, bemerkt: „The compositional unity of Gen 15 - from the literary, structural and thematic perspectives - marks it as the work of one single author." Für Ziemer, Abram - Abraham, 167, ist es jedoch ,eine Tatsache, dass Gen 15 eine mehrstufige Redaktionsgeschichte durchlaufen hat." Hinsichtlich der (Un)Einheitlichkeit des Kapitels betont Blum, „Verbindung,” 144 (Anmerkung): „,Relative Textkohärenz bleibt im Blick auf die Frage der Einheitlichkeit / Uneinheitlichkeit letztlich ein argumentum e silentio."

32 Blum, „Verbindung,” 142. Rolf Rendtorff, Das überlieferungsgeschichtliche Problem des Pentateuch, BZAW 147 (Berlin: de Gruyter, 1977), 164, versteht Gen 15 als „Haupttext“ einer deuteronomistischen Bearbeitungsschicht.

33 Ziemer, Abram - Abraham, 366; vgl. Rendtorff, Pentateuch, 136-141.

34 Vgl. Erich Zenger u.a., ${ }^{7}$ Einleitung in das Alte Testament (Stuttgart: Kohlhammer, 2008), 76-86.

35 Carr, Fractures, 3, postuliert, ,the book of Genesis [was] created out of earlier traditions (both written and oral) through a long process termed here 'transmission history'... our understanding of the present form of Genesis can be substantially deepened through historically informed analysis of its intense multivoiced character." Konrad Schmid, „Genesis in the Pentateuch," in The Book of Genesis: Composition, Reception, and Interpretation, hg. Craig A. Evans, Joel N. Lohr, und David L. Petersen, VTSup 152 (Leiden: Brill, 2012), 32, stellt fest: „Despite some introductory functions for the following books, Genesis also shows clear signs of having existed as a stand-alone literary unit for some portion of its literary growth."

36 Reinhard G. Kratz, „Der vor- und nachpriesterliche Hexateuch,” in Abschied vom Jahwisten: Die Komposition des Hexateuch in der jüngsten Diskussion, hg. Jan C. 
dargelegte verbale Verknüpfung manifestiert, dass Gen 15 (mit weiteren npTexten) und Gen 17, „kompositioneller ,Schluss-Stein“'37 der Genesis, unlösbar miteinander verflochten sind. Jenen Auslegern, die dem sichersten Ergebnis der Pentateuchkritik beipflichten, obliegt es, eine einleuchtende Erklärung dafür vorzulegen, dass die zwei Kapitel sich terminologisch in mehrfacher Hinsicht ergänzen und komplettieren. Im tradierten Text erweisen sich Gen 15 und Gen 17 als signifikante Abschnitte einer ,,absichtlich kombinierende[n] literarische[n] Konstruktion. “ 38

Die von der Pentateuchkritik aufgezeigten Spannungen und Widersprüche dürften dem für die Letztfassung Verantwortlichen nicht entgangen sein, doch er hat sie weder beseitigt noch retuschiert, sondern ,unausgeglichen aufgenommen." 39 Sein Textverständnis unterscheidet sich offensichtlich von dem unsrigen, denn seines entspricht nicht immer neuzeitlicher Logik. ${ }^{40}$ Gleichzeitig ist davon auszugehen, dass er sein opus magnum als sinnvoll komponiertes Ganzes gewertet hat, denn kein biblischer „Autor“ und kein Schriftsteller der Moderne kreiert einen Text, in dem die jeweils geltenden literarischen Kriterien ignoriert werden und ein Werk vorgelegt wird, das sich durch redundante Doppelungen und Widersprüche auszeichnet. Die sorgsame Setzung des Begriffs „Samen“ verdeutlicht sein Textverständnis, denn das Nomen gleicht einem roten Faden, der die gesamte Genesis durchzieht; auf diese Weise sind Urgeschichte, ${ }^{41}$

Gertz, Konrad Schmid, und Markus Witte, BZAW 315 (Berlin: de Gruyter, 2002), 295; Jan C. Gertz, Hg., ${ }^{5}$ Grundinformation Altes Testament: Eine Einführung in Literatur, Religion und Geschichte des Alten Testaments, UTB 2745 (Göttingen: Vandenhoek und Ruprecht, 2016), 243, behauptet: „Aufs Ganze gesehen sprechen die Argumente also weiterhin für die Annahme, dass es sich bei $\mathrm{P}$ um ein ehedem selbständiges Literaturwerk gehandelt hat.“ Schmid, „Genesis,” 34, ist der Auffassung, dass „Despite all the uncertainties of pentateuchal research, $P$ still remains a sufficiently safe assumption. Its texts probably formed a once independent literary entity that might have been written at the end of the sixth century BCE." Vgl. Rolf Rendtorff, Das Alte Testament: Eine Einführung (Neukirchen-Vluyn: Neukirchener Verlag, 1988), 166-173; Blum, Vätergeschichte, 229-285. Blum und Rendtorff deuten die priesterlichen Texte als Bearbeitungsschicht. Mit Blick auf das unterschiedliche Verständnis von „P“ bemerkt Römer, „Urkunden,“ 16: „Falls P sich wirklich als eine (vielschichtige) Bearbeitungsschicht erweisen sollte, wäre die Urkundenhypothese völlig ad acta zu legen und einer Ergänzungshypothese (kombiniert mit einer Fragmenten-Hypothese) der Vorrang zu geben.“

37 Ziemer, Abram - Abraham, 370.

38 Obgleich Fischer, „Penteuchforschung,” 614, hierbei den Pentateuch im Blick hat, trifft seine Aussage gleichermaßen auf die Genesis-Letztfassung zu.

39 Fischer, ,Pentateuchforschung,” 615.

40 Vgl. Zenger, u.a., Einleitung, 97-98.

41 Vgl. Jan C. Gertz, „The Formation of the Primeval History,” in The Book of Genesis: Composition, Reception, and Interpretation, hg. Craig E. Evans, Joel N. Lohr, und 
Erzelternerzählungen $^{42}$ und Josephgeschichte ${ }^{43}$ sinnvoll aneinandergereiht. $\mathrm{Ob}$ und inwieweit diese Großabschnitte in Beziehung zueinander stehen, wird in der Pentateuchforschung kontrovers diskutiert - ein Konsens ist nicht einmal schemenhaft in Sicht.

Der verbal fundierte und ,arithmo-logisch“ konzipierte Konnex, wie wir ihn bei diesen Mehrungsverheißungen beobachten, tritt allein in canonical shape zutage. Die bewusste Beschränkung, allein das Nomen „Samen“ zu analysieren, führt zu einzigartigen Einsichten in den literarischen Aufbau der Genesis. Weist dies möglicherweise hin auf eine „letzte ordnende Hand“? ?4 Diese Überlegungen führen hin zu der Arbeitshypothese, die dem Aufsatz zugrunde liegt: Zu einem uns unbekannten Zeitpunkt hat eine uns unbekannte Person nach uns (eher) unbekannten Kriterien die ihr vorliegenden Texte, seien sie noch mündlich oder

David L. Petersen, VTSup 152 (Leiden: Brill, 2012), 109, postuliert, ,the Primeval History of the Hebrew Bible is a closed, or we might say complete entity and was subsequently understood as such." Markus Witte, Die biblische Urgeschichte: Redaktionsund theologiegeschichtliche Beobachtungen zu Genesis 1,1-11,26, BZAW 265 (Berlin: de Gruyter, 1998), 146-50.

42 In den Erzelternerzählungen, so Rendtorff, Pentateuch, 29, tritt die „Sonderstellung der Josefgeschichte“ am klarsten hervor. Die Jakobgeschichte besteht aus „den JakobEsau-Geschichten ... und den Jakob-Laban-Geschichten,” die „kunstvoll miteinander verbunden sind“ (30). Im Abrahamzyklus zeigen sich „viele selbständige Überlieferungseinheiten, die keine ausdrücklichen Beziehungen zu ihrem Kontext haben,“ doch gleichzeitig gibt es einen ,inneren Zusammenhang[], der die ganze Abrahamüberlieferung durchzieht und als relativ geschlossene Einheit erscheinen läßt" (34).

43 Vgl. Carolin Paap, Die Josephsgeschichte Genesis 37-50: Bestimmungen ihrer literarischen Gattung in der zweiten Hälfte des 20. Jahrhunderts, EuroH 23/534 (Frankfurt am Main: Peter Lang, 1995); Horst Seebass, Genesis III: Josephsgeschichte (37,1-50,26), (Neukirchen-Vluyn: Neukirchener Verlag, 2000); Jürgen Ebach, Genesis 37-50, HThKAT (Freiburg: Herder, 2007); Franziska Ede, Die Josefsgeschichte: Literarkritische und redaktionsgeschichtliche Untersuchungen zur Entstehung von Gen 37-50, BZAW 485 (Berlin: de Gruyter, 2007); Michael V. Fox, „Joseph and Wisdom,” in The Book of Genesis: Composition, Reception, and Interpretation, hg. Craig A. Evans, Joel N. Lohr, und David L. Petersen, VTSup 152 (Leiden: Brill, 2012), 257, resümiert: „If one isolates in the Joseph Story motifs that are found in Wisdom Literature, then of course the story resembles that genre. But if one casts a wider net, the picture looks different. The story both overlaps Wisdom Literature ... and stands at a certain distance from it, because there are aspects of Joseph's wisdom that are of no interest in the didactic Wisdom Literature of Israel."

44 Vgl. Erhard Blum, „Gibt es die Endgestalt des Pentateuch?” in Congress Volume: Leuven 1989, hg. John A. Emerton, VTSup 43 (Leiden: Brill, 1991), 49. Mit Blick auf eine letzte ordnende Hand bemerkt Blum, gegen Ende stehe ,nicht der eine, alle Textelemente umgreifende Gestaltungswille, sondern eher ein Prozeß z.T. punktueller Fortschreibungen." 
bereits verschriftet gewesen, zu einem literarischen Ganzen zusammengefügt.

Damit die Übersichtlichkeit der 59-teiligen Tabelle gewahrt bleibt, sind nur die jeweils drei Belege in 13,14-17 / 28,13-15 gelistet. ${ }^{45}$ Nicht angeführt sind die ersten dreizehn Vorkommen $(1,112.122 .292 ; 3,152 ; 4,25 ; 7,3 ; 8,22 ; 9,9$; 12,7), die mittleren siebenundzwanzig $(15,3.5 .13 .18 ; 16,10 ; 17,72.8 .9 .10 .12 .19$; $19,32.34 ; 21,12.13 ; 22,172.18 ; 24,7.60 ; 26,3.43 .24 ; 28,4)$ und die letzten dreizehn $(32,13 ; 35,12 ; 38,8.92 ; 46,6.7 ; 47,19.23 .24 ; 48,4.11 .19)$ :

$\begin{array}{rrrll}13,15 & \ldots \text { Land ... dir und deinem } & \text { Samen } & \text { geben } & \\ 13,16 \mathrm{a} & \text { und ich werde deinen } & \text { Samen } & \text { machen } & \text { wie den Staub der Erde } \\ 13,16 \mathrm{~b} & \ldots \text {..wird auch deinen } & \text { Samen } & \text { zählen } & \\ \ldots & & & & \\ 28,13 \mathrm{~b} & \ldots \text { dir und deinem } & \text { Samen } & \text { geben } & \\ 28,14 \mathrm{a} & \text { dein } & \text { Samen } & \text { wird sein } & \text { wie der Staub der Erde } \\ 28,14 \mathrm{~b} & \text { gesegnet ... durch deinen } & \text { Samen } & & \end{array}$

Die komplexe Struktur fundiert auf dem Textgefüge, wie es uns in MT, Samaritanus und LXX (Vorlage) vorliegt. Ob dieser Sachlage verliert die These, ,it seems compelling that the main narrative in Gen $25 \mathrm{~B} ; * 27-33$ did not yet comprise the promises in 28:13b-14," "46 ungemein an Glaubwürdigkeit. In einem harmonischen „Zusammenklang“ von Urgeschichte, Erzelternerzählungen und Josephgeschichte, d.h., unter Einbeziehung aller Belege des Begriffs „Samen,“47 eignet Gen 13,16a und Gen 28,14a eine struktural einzigartige Stellung.

Die drei Textpaare $(13,16 \mathrm{a} / 28,14 \mathrm{a} ; 22,17 \mathrm{a} / 26,4 \mathrm{a} \alpha ; 17,7 \mathrm{a} / 17,19 \mathrm{~b})$ „unterteilen“ die Genesis in drei ineinander verschachtelte Großabschnitte (Gen 1,1-50,26 / 11,27-50,26 / 11,27-25,11). Jeder Teilabschnitt kündet Sinncharakter, ohne ihn verbal vorzutragen, da jede Texteinheit zwei theologisch

45 Erhard Blum, „Noch einmal: Jakobs Traum in Bethel - Genesis 28,10-22,“ in Rethinking the Foundations: Historiography in the Ancient World and the Bible: Essays in Honour of John Van Seters, hg. Steve L. McKenzie und Thomas Römer, BZAW 294 (Berlin: de Gruyter, 2000), 52, rechnet „in 28,13b-14 im wesentlichen mit einer (additiven) Einschreibung,“ wobei die Verheißungselemente „sachlich und kompositionell in einen späteren (exilischen) Kontext“ (54) gehören. Vgl. Schmid, „Genesis,” 34.

46 Erhard Blum, „The Jacob Tradition,” in The Book of Genesis: Composition, Reception, and Interpretation, hg. Craig A. Evans, Joel N. Lohr, und David L. Petersen, VTSup 152 (Leiden: Brill, 2012), 196. Hinsichtlich derart dezidierte Aussagen mahnt Schmid, „Genesis,” 27: „But if somebody is stating 'there can be no doubt' or 'all the evidence points to the fact,' then there usually is doubt, and not all the evidence points in the same direction" (Hervorhebung bei ihm).

47 Vgl. Ziemer, Abram - Abraham, 185: „Bloße Stichwortverknüpfungen sind prinzipiell nur im engeren Kontext überzeugend; in einem weiteren Kontext müssen schon die Übernahme einer größeren syntaktischen Einheit nachzuweisen sein." Bei den präsentierten verbalen Verknüpfungen handelt es sich nicht nur um „bloße Stichwortverknüpfungen," da jede einzelne auch "arithmo-logisch" ausgewogen konzipiert ist. 
signifikante Aussagen struktural hervorhebt. Im vorliegenden Textgefüge dient die Wiederaufnahme der leicht variierten Mehrungsverheißung von 13,16a in 28,14a dazu, die Abraham gegebene Zusage mit jener Verheißung zu vernetzen, die Jakob im Traum erhält. Diese Verflechtung ist terminologisch, thematisch und theologisch fundiert und beruht auf drei Gesichtspunkten: wortgleiche Metapher, derselbe Ort (Bethel) ${ }^{48}$ und die pointierte Positionierung. Verheißungsreden können kaum eindrücklicher miteinander verwoben sein.

Gemeinhin interpretiert man die Mehrungs- und Landverheißungen als sekundäre Ergänzungen. ${ }^{49}$ Indes zeitigt der jetzige Genesistext strukturierende Querverweise, die verschiedene Verheißungsreden miteinander verbinden. Die verbale und thematische Entsprechung von Gen 13,14-17 und 28,13-15 ist in der Forschung nicht unbekannt. Wann und von wem sind diese zwei Gottesreden verfasst und von wem sind sie wann in den jetzigen Kontext gestellt worden? Uns fehlt jedwede Information, um die Frage angemessen zu beantworten. Bei den beiden Verheißungsreden, „die kaum zum Urgestein der Erzählungen gehören, sondern spätere Kompositionselemente darstellen, "50 bilden 13,14-17 einen ,ersten Höhepunkt, “ der dann mit „Blick auf Isaak (Gen 26,3f.) und Jakob (Gen 28,13-15) wiederholt" ${ }^{\text {"51 }}$ wird. Sind Gen 13,14-17 und 28,13-15 ,in sich mehrschichtig ${ }^{652}$ und dienen als literarische Verbindung der „Abraham-LotErzählung und Jakobgeschichte zu einer kompositionellen Einheit" ${ }^{\text {"53? }}$ Oder sind sie redaktionellen Ursprungs ${ }^{54}$ und dienen dazu „rattacher le cycle de Jacob à celui d'Abraham (et à celui d'Isaac)“55? Haben wir es zu tun mit „Redactional Portions in Genesis Embedding the Book in the Pentateuch“"56 oder mit ,nachendredaktionelle[n] Ergänzungen“57? Gibt es in der Tat „deutliche Hinweise

48 In Genesis ist der Ortsname „Bethel“ dreizehn Mal belegt $\left(12,8^{2} ; 13,3^{2} ; 28,19\right.$; $31,13 ; 35,1.3 .6 .7 .8 .15 .16)$. Im fünften Text $(28,19)$ gibt Jakob der Stätte den Namen „Bethel und vormals war Luz der Name der Stadt,“ und in 35,6, dem fünfletzten Beleg, heißt es: „Und Jakob kam nach Luz, das im Lande Kanaan liegt, das ist Bethel.“

49 Vgl. Jacob Hoftijzer, Die Verheißungen an die drei Erzväter (Leiden: Brill, 1956), 17-23; 80f; Lothar Perlitt, Bundestheologie im Alten Testament, WMANT 36 (Berlin: de Gruyter, 1969), 68-77; John van Seters, Abraham in History and Tradition (New Haven: Yale University Press, 1975), 249-278); Rendtorff, Pentateuch, 40-65; Seebass, Josephsgeschichte, 233-239.

50 Gertz, Grundinformation, 276.

51 Gertz, Grundinformation, 277.

52 Rendtorff, Pentateuch, 110.

53 Nach Blum, Vätergeschichte, 297, ist diese Verbindung ,am ehesten in die Zeit zwischen dem politischen Ende des Nordreichs und dem Judas" zu datieren (Hervorhebung bei ihm).

54 Vgl. Jean-Louis Ska, Introduction à la lecture du Pentateuque: Clés pour l'interprétation des cinq premiers livres de la Bible (Brüssel: Éditions Lessius, 2000), 129.

55 Ska, Introduction, 134-135.

56 Bei Schmid, „Genesis,“ 37, ist das Zitat eine Überschrift.

57 Christoph Levin, Der Jahwist, FRLANT 157 (Göttingen: Vandenhoek \& Ruprecht, 
darauf, daß der Abschnitt 13,14-17 jünger ist als 28,13f und später in Anlehnung an diese Verse geschaffen wurde “58? Stammen sie aus der Feder eines vorexilischen Jahwisten ${ }^{59}$, der sein Werk zwischen „848 and $722^{\text {“60 }}$ verfasst hat ${ }^{61}$ ? Ist nur 13,14-16 ein „Nachtrag, “62 aber 28,13-15 auf gar keinen Fall? ${ }^{63}$ Die vorgetragenen Deutungsmodelle differieren deutlich, sind teilweise sogar unvereinbar. Ein signifikantes Indiz, dass die Diskussion um die Genese und strukturale Funktion der beiden Verheißungsreden mitnichten abgeschlossen ist. Sollte der tradierte Text aus verschiedenen „Vorstufen -mündlicher oder schriftlicher Art“ zusammengefügt sein, so dass die Vorstufen ,durch die Einordnung in den Gesamtkontext schwer erkennbar geworden"664 sind? Wenn K. Schmid anmerkt, „According to a quite common methodological consensus in diachronic biblical studies, it makes sense to begin with the (allegedly) later texts and then to proceed gradually to earlier ones," ${ }^{65}$ ist mit Blick auf die 59-teilige Tabelle zu fragen: Welche Kriterien stehen uns zur Verfügung, valide Kriterien, um entscheiden zu können, ob eine Belegstelle den „,(allegedly) later texts““ zuzurechnen ist oder aber den „earlier ones“? Die verbal und „arithmo-logisch“ ausgerichtete Balance, die Gen 13,16a zu 28,14a aufweist, zeigt allein der Endtext. Die referierten Ergebnisse verschiedener Ausleger, wann und warum die Verheißungsreden in 13,14-16 und 28,13-15 an ihren kanonischen Platz zu stehen kamen, manifestieren eine äußerst disparate Forschungslage. Da die äquidistante Struktur auf MT, Samaritanus und LXX (Vorlage) fußt, sei an B. S.

1993), 145 (13,14-15a) und 219 (28,13b-14.15a).

58 Ludwig Schmidt, „Väterverheißungen und Pentateuchfrage,” ZAW 104 (1992): 4.

$59 \mathrm{Zu}$ Gen 28,10-22 bemerkt Gordon J. Wenham, Genesis 16-50, WBC 2 (Waco: Word Books, 1994), 220-221: „So here, as elsewhere in Genesis, it seems likely that the last major editor of this story is J. His interests are particularly clear in the promises (vv 13-15)." Vgl. Gordon J. Wenham, Genesis 1-15, WBC 1 (Waco: Word Books, 1987), 294-295.

60 Richard E. Friedman, Who Wrote the Bible? (San Francisco: Harper, 1987), 87.

61 Im Hinblick auf deutlich differierende Datierungsvorschläge in der Forschung bemerkt Ronald Hendel, „Historical Context,” in The Book of Genesis: Composition, Reception, and Interpretation, hg. Craig A. Evans, Joel N. Lohr, und David L. Petersen, VTSup 152 (Leiden: Brill, 2012), 51: „The weak connective tissue of many recent arguments about the historical context of Genesis is a reflection of the paucity of historical data, which is - alas - the condition of historians of Levantine antiquity. Cognizance of the limits of our evidence should constrain our historical speculations."

62 Horst Seebass, Genesis II/1: Vätergeschichte I $(11,27-22,24)$ (Neukirchen-Vluyn: Neukirchener Verlag, 1997), 39.

63 Horst Seebass, Genesis II/2: Vätergeschichte II (23,1-36,43) (Neukirchen-Vluyn: Neukirchener Verlag, 1999), 322: Dass manche Gen 28,13-15 „als Nachtrag erklären ... liegt an m.E. viel zu allgemeinen Theorien über die Funktion der verschiedenen Verheißungen in der Genesis, nicht aber an sicheren Kontextbeobachtungen."

64 Georg Fischer, „Wege aus dem Dunkel: Ein Beitrag zur Pentateuchkrise,” BN 99 (1999): 7.

65 Schmid, „Genesis,” 33. 
Childs Aussage, ,easy diachronic reconstructions ... fracture the witness of the whole,"66 erinnert. Sollte ,the witness of the whole“" nach dem Zufallsprinzip entstanden sein, verdient die Tabelle keinerlei Beachtung. Verdankt sie ihre Existenz jedoch wohlbedachter Kompositionstechnik, gebührt ihr ungeteilte Aufmerksamkeit.

Auch die folgenden Tabellen unterstreichen die verbal und ,arithmologisch“ konzipierte Verflechtung von Gen 13,14ff ${ }^{67}$ mit 28,13ff. Bei seiner Rückkehr aus Ägypten zieht Abraham ,vom Negeb bis nach Bethel, bis zu dem Ort, an dem er anfangs gezeltet hatte, zwischen Bethel und Ai““ $(13,3)$. In der ersten Gottesrede, die nach der Trennung von Lot an ihn ergeht, heißt es: „Hebe doch deine Augen auf und schaue von der Stätte, an der du bist, nach Norden und Süden, nach Osten und Westen, denn das ganze Land, das du schaust, dir gebe ich es und deinem Samen für immer“ (13,14b-15). In 28,13b-14a erhält Jakob die Zusage: „Das Land, auf dem du liegst, dir gebe ich es und deinem Samen, und dein Samen wird sein wie der Staub der Erde, und du wirst dich ausbreiten nach Westen und Osten, nach Norden und Süden." Ungeachtet ihrer semantischen Nuancierung scheinen auch die Nomina ים "Meer; Westen“ (Gen נגב 1,10.22.26.28; 9,2;12,8;13,14;14,3;22,17;28,14;32,13;41,19;49,13) und „Negeb; Süden“ (12,9; 13,1.3.14; 20,1; 24,62; 28,14) Teile der Genesis zu gliedern. In der 13-teiligen ים-Tabelle steht Gen 13,14 an siebter Stelle, d.h. im Mittelpunkt; in der 7-teiligen נגב-Tabelle hat 13,14 erneut die zentrale Position inne, dieses Mal jedoch die vierte, während 28,14 nunmehr den siebten Platz einnimmt.

Auch das nachstehende Diagramm lässt sorgsame Textgestaltung vermuten. Gott offenbart sich den Erzvätern bei / in Bethel; in den Verheißungsreden weist er Abraham und Jakob auf die vier Himmelsrichtungen hin; ,schaue ... nach Norden und Süden, nach Osten und Westen, denn das ganze Land, das du schaust, dir gebe ich es und deinem Samen ...“ (13,14-15) / (28,13b-14a), das Land ... dir gebe ich es und deinem Samen ... du wirst dich ausbreiten nach Westen und Osten, nach Norden und Süden." $\mathrm{Zu}$ beachten ist die chiastisch anmutende Anordnung der Himmelsrichtungen (Osten / Westen // Westen / Osten). Gen 13,14-15 nennt sie vor der Landverheißung und 28,13-14 danach. ${ }^{68}$ Die Landverheißung לד אתננה ולזרעך, dir gebe ich es und deinem Samen“ $(13,15 ; 28,13)$, die so formuliert im Pentateuch nur hier vorliegt, bildet das Herzstück des 6-teiligen Diagramms:

66 Brevard S. Childs, Old Testament Theology in a Canonical Context (Minneapolis: Fortress Press, 1985), 11.

${ }^{67}$ Ha, Genesis 15, 201, versteht „Gen. 12:4b-13:18 ... as a prelude to the rest of the Pentateuch."

68 Laut Blum, Vätergeschichte, 291, dient die Nennung der vier Himmelrichtungen in Gen 13 ,der Gestaltung der Landverheißung“ und in Gen 28 ,als Weiterführung auf die Mehrungsverheißung.“ 
Warning, “Gedanken zu dem Nomen," OTE 30/3 (2017): 852-873

\begin{tabular}{cc}
\hline 13,14 & Norden und Süden \\
13,14 & Osten und Westen \\
13,15 & $\ldots \quad$ das Land, ... dir gebe ich es und deinem Samen \\
28,13 & $\ldots \quad$ das Land, ... dir gebe ich es und deinem Samen \\
28,14 & Westen und Osten \\
28,14 & Norden und Süden
\end{tabular}

Augenscheinlich enthält der jetzige Text mehrere Momente, welche die Gottesreden in 13,14-16 / 28,13-15 miteinander verbinden.

\section{B GENESIS 11,27-50,26}

Die Erzelterngeschichten schildern „die Anfänge des Volkes Israel in der literarischen Form der Familienerzählung, “69 die nicht mit der „Esau-Toledot" in Gen 36 endet, sondern den Erzählfaden in der „Jakob-Toledot“ (Gen 37-50) weiterführt. ${ }^{70}$ Daher wird man die Josephgeschichte nicht a priori von den vorangehenden Erzählungen trennen können, denn „die Verknüpfungen zwischen Erzeltern- und Josefsgeschichte" zeigen sich in den ,Wiederholungen' von Worten, Motiven und Themen aus Gen 12-36 in 37-50.“71 In der Diskussion um die Interdependenz von Erzelternerzählungen und Josephgeschichte ist eine communis opinio nicht in Sicht, doch aufgrund der inhaltlichen und terminologischen Verquickung werden Gen 12-36 plus 37-50 hier als literarische Erzähleinheit verstanden. In diesem Großabschnitt kommt das Nomen „Samen“ siebenundvierzig Mal vor $(12,7 ; 13,15.162 ; 15,3.5 .13 .18 ; 16,10$; 17,72.8.9.10.12.19; 19,32.34; 21,12.13; 22,172.18; 24,7.60; 26,3.43.24; $28,4.13 .142 ; 32,13 ; 35,12 ; 38,8.92 ; 46,6.7 ; 47,19.23 .24 ; 48,4.11 .19)$. Hierbei fällt auf, dass es zwar in der Terach-Toledot $(11,27-25,11)$, Isaak-Toledot $(25,19-35,28)$ und Jakob-Toledot $(37,2-50,26)$ vorliegt, jedoch nicht ein einziges Mal in der Ismael-Toledot $(25,12-19)$ und der Esau-Toledot $(36,1-8.9-43)$. Bei den „Nebenlinien“ ist allein von ihren „Söhnen“ die Rede.

In Gen 22,17a a schwört Gott bei sich selbst, ,ich werde deinen Samen reichlich mehren wie die Sterne des Himmels, “72 und in 26,4a $\alpha$ erhält Isaak die eidliche Zusicherung, ,,ich werde deinen Samen mehren wie die Sterne des Himmels. "“73 Dass die Gottesreden in 26,3b-5 und 22,15-18 einander inhaltlich

69 Gertz, Grundinformation, 276.

70 Vgl. Naomi A. Steinberg, ,The World of the Family in Genesis," in The Book of Genesis: Composition, Reception, and Interpretation, hg. Craig A. Evans, Joel N. Lohr, und David L. Petersen, VTSup 152 (Leiden: Brill, 2012), 282: „Analysis of structure and theme in Genesis in its final form reveals a focus on kinship and family as a divine concern from creation through the sojourn of Jacobs's family in Egypt.“

71 Ebach, Genesis 37-50, 680.

72 Blum, Vätergeschichte, 390, versteht die Gottesrede in Gen 22,15ff als ,deutliches Gegenstück zu der in 12,2f ... und markiert mit dieser Rahmung den Abschluß der Abrahamgeschichte in Gen 22.“

73 Nach Schmidt, „Väterverheißungen,“ 10, gehören die „Verheißungen in 22,15-18; 26,3b-5 und 26,24 ... zu einer sehr späten Bearbeitung im Umkreis der Endredaktion 
gleichen, ist hinlänglich bekannt ${ }^{74}$ - unbekannt ist die augenfällige ,arithmologische“ Balance, die 22,17a $\alpha$ in Beziehung setzt zu 26,4a . In der 47-teiligen Tabelle hat 22,17a $\alpha$ den einundzwanzigsten Platz inne und 26,4a den einundzwanzigletzten. Dient die pointierte Placierung der Metapher ,wie die Sterne des Himmels" dazu, die Verflechtung von $22,15-18^{75}$ und 26,3b-5 und damit den Konnex des Abrahamzyklus und der (vermeintlich) „selbständige[n] Isaakgeschichte" 76 in Gen 26 strukturell hervorzuheben? ${ }^{77}$ Die passgenaue Positionierung von 22,17a $\alpha$ und 26,4a $\alpha$ lässt Zweifel aufkommen an der These, „Daß Kap. 26 im Kontext an verfehlter Stelle steht, ist opinio communis. “" ${ }^{" 78}$ Woher wissen wir, dass „die Verheißungsrede in Gen 22,15-18 überlieferungsgeschichtlich von der älteren Erzählung in Gen $22^{\text {“79 }} \mathrm{zu}$ unterscheiden ist? Die Aussage dürfte kaum auf dem vorliegenden Text, ,the only fact available to us ... in all its complexity," 80 beruhen, sondern wohl eher auf persönlichen Prämissen. Der von E. Blum vorgetragenen These, „Es gibt in etwa so viele Endgestalten wie Textzeugen, " ${ }^{\circ 81}$ ist zwar beizupflichten; doch mit Blick auf die Genesis-Letztfassung (d.h., bezogen auf die Setzung des Wortes „Samen“) gilt zu bedenken, dass jede hier vorgetragene äquidistante Struktur auf drei Textzeugen basiert.

Im Rahmen von Gen 11,27-50,26 dienen 22,17a $\alpha$ und 26,4a $\alpha$ in erster Linie dazu, die an Abraham ergangene Verheißungsrede in 22,15-18 mit der

des Pentateuch“; Carr, Fractures, 153, sieht 22,15-18 und 26,3b $\beta$ als ,,marked secondary additions to that context."

74 Vgl. u.a. Rendtorff, Pentateuch, 161, rechnet Gen 22,15-18, „nun auch zu den Textstücken, durch welche die drei Vätergeschichten miteinander verbunden und zu einem Ganzen zusammengefügt warden.“ Blum, Vätergeschichte, 363-365; David M. Carr, „Scribal Processes of Coordination / Harmonization and the Formation of the First Hexateuch(s)," in The Pentateuch: International Perspectives on Current Research, hg. Thomas B. Dozeman, Konrad Schmid, und Baruch J. Schwartz, FAT 78 (Tübingen: Mohr Siebeck, 2011), 77.

75 Blum, Vätergeschichte, 367, weist 22,15-18 und 26,3b-5 der gleichen Hand zu wie 15,1-6. Für Rendtorff, Pentateuch, 59, bilden Gen 22,15-18 „den betonten Abschluß der Abrahamgeschichte."

76 Rendtorff, Pentateuch, 31.

77 Vgl. John A. Emerton, „The Origin of the Promises to the Patriarchs in the Older Sources of the Book of Genesis," VT 32 (1982): 14.

78 Seebass, Vätergeschichte II, 278. Wenham, Genesis 16-50, 186, stellt die Frage: „Is there any logic to its being placed here in that cycle, rather than right at the beginning? Or must we concur with Skinner ... that it is 'a misplaced appendix to the history of Abraham'?" Vgl. Dieckmann, Isaak, 317-324.

79 Blum, Vätergeschichte, 320; vgl. Carr, Fractures, 153.

80 R. Norman Whybray, Introduction to the Pentateuch (Grand Rapids: Eerdmans, 1995), 27 (Hervorhebung bei ihm).

81 Nach Blum, „Endgestalt,” 46, ist auch „,die Endgestalt ... keine gegebene Entität, sondern existiert nur als Hypothese (und d.h. de facto: in der Pluralität vieler Hypothesen).“ 
ersten an Isaak gerichteten Gottesrede in 26,3b-5 zu verflechten. Die sorgsame Setzung der Metapher ,wie die Sterne des Himmels“ indiziert, jene Person, der wir die Erzählungen von Abraham und Isaak, Jakob und Joseph verdanken, scheint den Passus als literarische „Einheit“" verstanden zu haben. Um die Übersichtlichkeit der Tabelle zu wahren, sind die ersten achtzehn Belege (12,7; $13,15.162 ; 15,3.5 .13 .18 ; 16,10 ; 17,72.8 .9 .10 .12 .19 ; 19,32.34)$ und die letzten achtzehn $(26,24 ; 28,4.13 .142 ; 32,13 ; 35,12 ; 38,8$ 92; 46,6.7; 47,19.23.24; 48,4.11.19) nicht aufgeführt:

\begin{tabular}{|c|c|c|c|}
\hline 21,12 & ... soll dein & Samen & benannt werden \\
\hline 21,13 & denn er ist dein & Samen & \\
\hline $22,17 \mathrm{a}$ & ich werde deinen & Samen & ... wie die Sterne des Himmels \\
\hline $22,17 b$ & und dein & Samen & soll besitzen die Tore ihrer Feinde \\
\hline 22,18 & durch deinen & Samen & sollen alle Völker ... \\
\hline 24,7 & ... deinem & Samen & werde ich dieses Land geben \\
\hline 24,60 & und dein & Samen & soll besitzen die Tore ihrer Hasser \\
\hline 26,3 & dir und deinem & Samen & werde ich alle diese Länder geben \\
\hline $26,4 \mathrm{a} \alpha$ & ich werde deinen & Samen & ... wie die Sterne des Himmels \\
\hline $26,4 \mathrm{a} \beta$ & ich werde deinem & Samen & alle diese Länder geben \\
\hline $26,4 b$ & und mit deinem & Samen & werden sich segnen ... \\
\hline
\end{tabular}

Der zentrale Text der 47-teiligen Tabelle lautet: „JHWH, der Gott des Himmels ... der zu mir geredet und mir geschworen hat, ,deinem Samen werde ich dieses Land geben’..." ${ }^{82}$ (24,7). Die einzige als Schwur formulierte Landverheißung, in der sich der Empfänger expressis verbis auf Gottes eidliche Zusage beruft, steht im Mittelpunkt. Die prononcierte Positionierung und die Tatsache, dass Gen 24 nahtlos in jede „Samen“-Struktur eingebunden ist, stellen die Hypothese, Gen 24 sei ,in den bereits priesterlich edierten *Pentateuch" ${ }^{\text {"83 }}$ eingeschrieben, ernsthaft infrage. Die Tabelle betont einerseits die Interdependenz der Verheißungsreden in 22,15-18 und 26,3b-5, andrerseits wird Gen 24,7 durch die Textgestaltung zum Herzstück, denn die als Schwur formulierte Landverheißung steht im Zentrum.

\section{GENESIS 11,27-25,11}

„Um einen Text verstehen zu können, sollte man wissen, wo/wie er anfängt und wo/wie er aufhört. “ ${ }^{84}$ Blums Aussage, der vorbehaltlos zuzustimmen ist, ,can be

82 Zu der Frage, „ob 24,7a einen dem נשבע entsprechenden Bezugstext hat,“ erklärt Blum, Vätergeschichte, 376: „Zwar wird in 15,18 nicht der Ausdruck נשבע gebraucht, sondern כרת ברית,“ doch „schon in der erzählenden Überlieferung [sind] ,Eid' und ,Bund' nahezu austauschbar.“"

83 Blum, „Verbindung,” 142; Rendtorff, Pentateuch, 59 A. versteht Gen 23 und Gen 24 als „eine Art Anhang oder Nachtrag zu der schon weitgehend als Einheit ausgestalteten Abrahamgeschichte."

84 Erhard Blum, „Pentateuch - Hexateuch - Enneateuch? Oder: Woran erkennt man ein literarisches Werk in der Bibel?" in Les dernières rédactions du Pentateuque, de 
regarded as a central question for current pentateuchal research. ${ }^{* 85}$ Versteht man die „Toledot-Formel als Gliederungssignal“"86 der Genesis, sind Anfang und Ende der Abrahamgeschichte unschwer festzustellen. ${ }^{87}$ In dem Abschnitt liegt das Nomen „Samen“ fünfundzwanzig Mal vor, siebenmal in Gen 17 (V. 72.8.9.10.12.19), neunmal davor $(12,7 ; 13,15.162 ; 15,3.5 .13 .18 ; 16,10)^{88}$ und neunmal danach $(19,32.34 ; 21,12.13 ; 22,172.18 ; 24,7.60)$. Somit steht außer Frage, den Kern der 25-teiligen Tabelle bildet Gen 17. In 17,7a.19b begegnet die Wendung הקים ברית ... לברית עולם, einen Bund aufrichten ... als ewigen Bund“ - im Tanach ist sie nur zweimal bezeugt. Vergleicht man 17,7a (,ich werde aufrichten meinen Bund zwischen mir und dir und deinem Samen nach dir in ihren Generationen als ewigen Bund“) mit 17,19b (,,ich werde aufrichten meinen Bund mit ihm als ewigen Bund für seinen Samen nach ihm“) ist festzuhalten: Die an Abraham und seinen (noch nicht geborenen) Sohn Isaak gerichtete Bundeszusage dient in Gen 17 als literarischer Rahmen; gleichzeitig fassen die zwei Bundeszusagen die Kernbotschaft des Kapitels subtil zusammen. Mit Blick auf 17,7 konstatiert H. Sebass, dass in

dem nüchternen Satz von V7 ... die vielleicht wichtigste theologische Wahrheit der altisraelitischen Geschichte als Abmachung Gottes eingefangen [ist]. Die nicht zu kündigende Abmachung Gottes mit seinem Volk in Abraham bezeichnet zweifellos eines der größten Wunder und Geheimnisse der Weltgeschichte bis heute. ${ }^{89}$

Wenn bei Isaak in V. 19 der Ausdruck ,als ewigen Bund“ von V. 7 wiederkehrt, „kann dies nur bedeuten, daß V.19-21 als verbindliche Interpretation der Zusage von V.7f verstanden sein wollen. ${ }^{\text {"90 }}$ Durch die Wiederaufnahme

l'Hexateuque et de l'Ennéateuque, hg. Thomas Römer und Konrad Schmid, BETL 203 (Leuven: University Press, 2007), 67.

85 Kratz, „Pentateuch,” 31.

86 Hieke, Genealogien, 45. Die Setzung der dreizehn Belege des Wortes תולדות „Toledot" $(2,4 ; 5,1 ; 6,9 ; 10,1.32 ; 11,10.27 ; 25,12.13 .19 ; 36,1.9 ; 37,2)$ kann ebenfalls als literarisches Gestaltungsmittel gedeutet werden. In der 13-teiligen Tabelle nimmt die Nominalform לתולדתם,nach ihren Toledot,” die in Genesis nur zweimal vorkommt, die fünfte $(10,32)$ und fünfletzte $(25,13)$ Stelle ein. Im Mittelpunkt, an siebter Stelle, steht die Terach-Toledot in 11,27-25,11.

87 Vgl. Hieke, Genealogien, 242: „Das Toledot-System gliedert das Buch Genesis in seiner Endgestalt in Großabschnitte, die sich wie die Kapitel eines Romans an den 'Nahtstellen' leicht überlappen."

88 In Gen 15,18 versteht Gertz, „Abraham,” 66, die „Formulierung כרת ברית את mit Jahwe als Subjekt [als] den terminus technicus für den ,Bundesschluss' am Sinai / Horeb“ (vgl. Ex 34,27; Dtn 5,3; 28,69; 29,13; 31,16). Beim Bundesschluss in Ex 24,8

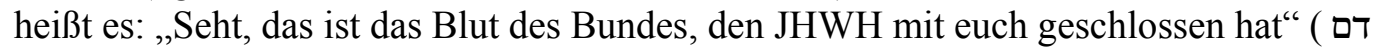
(הברית אשר כרת יי עמכם עם wird auch in Dtn 4,23; 5,2; 9,9; 29,11.24 verwendet.

89 Seebass, Vätergeschichte 1, 104.

90 Blum, Vätergeschichte, 422. 
des Gedankens von V. 7a in V. 19b ist nicht nur Gen 17 an sich sinnvoll gegliedert, sondern in der 25-teiligen äquidistanten Struktur, sie stellt alle Vorkommen des Begriffs zusammen, hat 17,7a den zehnten und 17,19b den zehnletzten Platz inne. Damit die Tabelle übersichtlich bleibt, werden nur die sieben Belege aus Gen 17 gelistet, die ersten neun $(12,7 ; 13,15.162$; $15,3.5 .13 .18 ; 16,10)$ und die letzten neun $(19,32.34 ; 21,12.13 ; 22,172.18$; $24,7.60)^{91}$ sind nicht angeführt:

$\begin{array}{lrll}17,7 \mathrm{a} & \text { aufrichten } \mathrm{m} . \text { Bund zwischen ... deinem } & \text { Samen } & \text { nach dir } \\ 17,7 \mathrm{~b} & \text { um dein Gott zu sein und der deines } & \text { Samen } & \text { nach dir } \\ 17,8 & \text { dir und deinem } & \text { Samen } & \text { nach dir ... } \\ 17,9 & \text { du und dein } & \text { Samen } & \text { nach dir ... } \\ 17,10 & \text { zwischen mir und euch und deinem } & \text { Samen } & \text { nach dir } \\ 17,12 & \text { die nicht von deinem } & \text { Samen } & \text { sind } \\ 17,19 \mathrm{~b} \text { aufrichten } \mathrm{m} . \text { Bund mit ihm ... für seinen } & \text { Samen } & \text { nach ihm }\end{array}$

Die gewichtige theologische Wahrheit, die unverbrüchliche Bundeszusage Gottes für Abraham und Isaak, wird in doppelter Hinsicht struktural unterstrichen, einmal im Kapitel per se und zweitens geben Gen 17,7a und Gen 17,19b der gesamten Abrahamgeschichte theologische Signifikanz. Bei der 25teilige Tabelle, die aus p-Texten und np-Texten besteht, vernimmt man gewissermaßen einen einzigartigen, einen harmonischen ,Einklang. “92

91 Folgende Beobachtung bestätigt die Plausibilität der Tabelle. Streicht man Gen 19,32.34, zeigt sich eine 23-teilige Struktur, die auf Abrahams „Samen“ abzielt (12,7; $\left.13,15.16^{2} ; 15,3.5 .13 .18 ; 16,10 ; 17,7^{2} .8 .9 .10 .12 .19 ; 21,12.13 ; 22,17^{2} .18 ; 24,7.60\right)$. Im Pentateuch kommt der Ausdruck ול)זרעך אחריך,, (und für) deinen Samen nach dir“ fünfmal vor $\left(17,7^{2} .8 .9 .10\right)$ - den fünf Texten gehen neun Belegstellen voran und neun folgen.

92 Dieses Phänomen offenbart sich auch dann, wenn man alle Belege des Begriffs ברית „Bund“ in Gen 11,27-25,11 zusammenstellt. Das Nomen, es scheint das Leitwort in Gen $17 \mathrm{zu}$ sein, kommt in der Abrahamgeschichte siebzehn Mal vor (14,13; 15,18; 17,2.4.72.9.10. 11.132 $14.19^{2} .21$; 21,27.32); allein dreizehn Mal in Gen 17, d.h., häufiger als irgendwo sonst im Tanach. In der 13-teiligen Tabelle nimmt der Satz „und ich will aufrichten meinen Bund zwischen dir und mir" (17,7a $\alpha)$ die dritte und ,ich will aufrichten meinen Bund mit ihm" (17,19a $\alpha$ ) die drittletzte Position ein. In der 17teiligen Struktur, zusammengesetzt aus vier np-Texten und dreizehn p-Texten, steht $17,7 \mathrm{a} \alpha$ an fünfter und 17,19b $\alpha$ an fünfletzter Stelle. Folglich eignet den beiden Texten im Abrahamzyklus eine einzigartige Stellung. In der 27-teiligen „Samen“-Tabelle und auch in der 17-teiligen „Bund“-Tabelle strukturieren die zwei Aussagen nicht nur Gen 17 an sich, sondern verleihen Gen 11,27-25,11 eine theologisch relevante Struktur. Hervorzuheben ist, dass diese verbal und „arithmo-logisch“ fundierten Querbezüge allein in canonical shape zutage treten, d.h., in der aus p-Texten und np-Texten zusammengefügten „Endgestalt.“

Im Pentateuch kommt der Begriff הקים ברית הeinen Bund aufrichten“ zehnmal vor (Gen 6,18; 9,9.11.17; 17,7.19.21; Ex 6,4; Lev 26,9; Dtn 8,18), neunmal in p-Texten und ein einziges Mal in Dtn 8,18. Auch hier bilden Gen 17,7a und 17,19b den strukturalen / theologischen Kern, denn in der 10-teiligen äquidistanten Struktur steht Gen 17,7a an 
Die drei זרע-Strukturen lassen sechs „Fixpunkte“(13,16a / 28,14a; 22,17a / 26, 4aa; 17,7a / 17,19b) aufleuchten, von denen jeder fest im tradierten Text verankert ist. Eine „diachrone Thesenbildung“ muss sich laut Seebass „stets daran bewähren, daß sie die Endgestalt besser erklärt als ohne sie. “93 In der künftigen Genesisforschung sollte der ausgewogene „Dreiklang“ der drei זרעStrukturen beachtet und angemessen zur Kenntnis genommen werden.

\section{SCHLUSSGEDANKEN}

Liest man das erste Buch der Bibel unter dem Blickwinkel ausgewählter Mehrungsverheißungen, zeigt sich eine literarische Konstruktion, in der diverse „priester(schrift)liche“ und nicht-priesterliche Texte $\mathrm{zu}$ einem terminologisch ausgerichteten Ganzen zusammengefügt sind. Carr fordert eine sorgsam durchgeführte synchrone Textanalyse - sie liegt hier vor, bewusst beschränkt auf das Nomen „Samen.“ Was fehlt ist „methodologically controlled reflection on how that form was produced." 94 Die verbal fundierte und ,arithmo-logisch" konzipierte Verflechtung von Gen 13,16a / 28,14a; 22,17a / 26,4a $\alpha$ und 17,7a / $17,19 \mathrm{~b}$ bei den Mehrungsverheißungen zeitigt allein canonical shape. Das kanonische Textgefüge richtet den Blick auf einen Aspekt einer ,Theologie der Genesis“: „The promise theme is probably the most prominent element in Genesis which has a significance of its own. “95

\section{BIBLIOGRAPHIE}

Anbar, Moshe. „La 'Reprise.'“ VT 38 (1988): 385-398.

Blum, Erhard. Die Komposition der Vätergeschichte. WMANT 57. Neukirchen-

Vluyn: Neukirchener Verlag, 1984. . „Gibt es die Endgestalt des Pentateuch?“ Seiten 46-57 in Congress Volume:

Leuven 1989. Hg. John A. Emerton. VTSup 43. Leiden: Brill, 1991. . „Noch einmal: Jakobs Traum in Bethel - Genesis 28,10-22.” Seiten 33-54

in Rethinking the Foundations. Historiography in the Ancient World and the Bible: Essays in Honour of John van Seters. Hg. Steve L. McKenzie und Thomas Römer. BZAW 294. Berlin: de Gruyter, 2000. . „Die literarische Verbindung von Erzvätern und Exodus: Ein Gespräch mit neueren Endredaktionshypothesen." Seiten 119-156 in Abschied vom Jahwisten: Die Komposition des Hexateuch in der jüngsten Diskussion. Hg. Jan C. Gertz, Konrad Schmid und Markus Witte. BZAW 315. Berlin: de Gruyter, 2002. . „Pentateuch - Hexateuch - Enneateuch? Oder: Woran erkennt man ein literarisches Werk in der Bibel?" Seiten 67-97 in Les dernières rédactions du Pentateuque, de l'Hexateuque et de l'Ennéateuque. Hg. Thomas Römer und Konrad Schmid. BETL 203. Leuven: University Press, 2007. . „The Jacob Tradition.” Seiten 181-211 in The Book of Genesis:

fünfter und $17,19 \mathrm{~b}$ an fünfletzter Stelle.

93 Seebass, Josephsgeschichte, 217.

94 Carr, Fractures, viii.

95 Schmid, „Genesis,” 30. 
Composition, Reception, and Interpretation. Hg. Craig E. Evans, Joel N. Lohr, und David L. Petersen. VTSup 152. Leiden: Brill, 2012.

Buber, Martin. „Leitwortstil in den Erzählungen des Pentateuch.“ Seiten 1131-1149 in Schriften zur Bibel, 2. München: Kösel Verlag, 1964.

Carr, David M. Reading the Fractures of Genesis: Historical and Literary Approaches. Louisville: Westminster John Knox Press, 1996.

. "Scribal Processes of Coordination/Harmonization and the Formation of the First Hexateuch(s)." Seiten 63-83 in The Pentateuch: International Perspectives on Current Research. Hg. Thomas B. Dozeman, Konrad Schmid, und Baruch J. Schwartz. FAT 78. Tübingen: Mohr Siebeck, 2011.

Childs, Brevard S. Old Testament Theology in a Canonical Context. Minneapolis: Fortress Press, 1985.

De Pury, Albert. „Gottesname, Gottesbezeichnung und Gottesbegriff. 'Elohim als Indiz zur Entstehungsgeschichte des Pentateuch." Seiten 25-47 in Abschied vom Jahwisten: Die Komposition des Hexateuch in der jüngsten Diskussion. Hg. Jan C. Gertz, Konrad Schmid, und Markus Witte. BZAW 315. Berlin: de Gruyter, 2002.

Dieckmann, Detlef. Segen für Isaak: Eine rezeptionsästhetische Auslegung von Genesis 26 und Kotexten. BZAW 329. Berlin: de Gruyter, 2003.

Ebach, Jürgen. Genesis 37-50. HThKAT. Freiburg: Herder, 2007.

Ede, Franziska. Die Josefsgeschichte: Literarkritische und redaktionsgeschichtliche Untersuchungen zur Entstehung von Gen 37-50. BZAW 485. Berlin: de Gruyter, 2016.

Emerton, John A. „The Origin of the Promises to the Patriarchs in the Older Sources of the Book of Genesis." VT 32 (1982): 14-32.

Fischer, Georg. „Wege aus dem Dunkel: Ein Beitrag zur Pentateuchkrise.” BN 99 (1999): 5-7.

. „Zur Lage der Pentateuchforschung.“ ZAW 115 (2003): 608-616.

Fox, Michael V. „Joseph and Wisdom.” Seiten 231-262 in The Book of Genesis: Composition, Reception, and Interpretation. Hg. Craig A. Evans, Joel N. Lohr und David L. Petersen. VTSup 152. Leiden: Brill, 2012.

Friedman, Richard E. Who Wrote the Bible? New York: HarperCollins, 1987.

Gertz, Jan C. „Abraham, Mose und der Exodus: Beobachtungen zur Redaktionsgeschichte von Gen 15." Seiten 63-81 in Abschied vom Jahwisten: Die Komposition des Hexateuch in der jüngsten Diskussion. Hg. Jan C. Gertz, Konrad Schmid, und Markus Witte. BZAW 315. Berlin: de Gruyter, 2002. . „The Formation of the Primeval History.” Seiten 107-136 in The Book of Genesis: Composition, Reception, and Interpretation. Hg. Craig E. Evans, Joel N. Lohr und David L. Petersen. VTSup 152. Leiden: Brill, 2012. . Grundinformation Altes Testament: Eine Einführung in Literatur, Religion und Geschichte des Alten Testaments. 5th ed. UTB 2745. Göttingen: Vandenhoek \& Ruprecht, 2016.

Ha, John. Genesis 15: A Theological Compendium of Pentateuchal History. BZAW 181. Berlin: de Gruyter, 1989.

Hendel, Ronald. „Historical Context.“ Seiten 51-81 in The Book of Genesis: Composition, Reception, and Interpretation. Hg. Craig E. Evans, Joel N. Lohr und David L. Petersen. VTSup 152. Leiden: Brill, 2012. 
872 Warning, “Gedanken zu dem Nomen,” OTE 30/3 (2017): 852-873

Hieke, Thomas. Die Genealogien der Genesis. HBSt 39. Freiburg: Herder, 2003. Hoftijzer, Jacob. Die Verheißungen an die drei Erzväter. Leiden: Brill, 1956.

Jacob, Benno. Das Buch Genesis. Stuttgart: Calwer Verlag, 2000.

Kratz, Reinhard G. „Der vor- und nachpriesterliche Hexateuch.“ Seiten 295-323 in Abschied vom Jahwisten: Die Komposition des Hexateuch in der jüngsten Diskussion. Hg. Jan C. Gertz, Konrad Schmid, und Markus Witte. BZAW 315. Berlin: de Gruyter, 2002. . „The Pentateuch in Current Research: Consensus and Debate.” Seiten 31-61 in The Pentateuch: International Perspectives on Current Research. Hg. Thomas B. Dozeman, Konrad Schmid, und Baruch J. Schwartz. FAT 78. Tübingen: Siebeck Mohr, 2011.

Levin, Christoph. Der Jahwist. FRLANT 157. Göttingen: Vandenhoek \& Ruprecht, 1993.

McEvenue, Sean E. The Narrative Style of the Priestly Writer. AnBib 50. Rom: Biblical Institute Press, 1971.

Otto, Eckart. „Forschungen zum nachpriesterlichen Pentateuch.“ TRu 67 (2002): 125 155.

Paap, Carolin. Die Josephsgeschichte Genesis 37-50: Bestimmungen ihrer literarischen Gattung in der zweiten Hälfte des 20. Jahrhunderts. EuroH 23/534. Frankfurt a.M: Peter Lang, 1995.

Perlitt, Lothar. Bundestheologie im Alten Testament. WMANT 36. Berlin: de Gruyter, 1969.

Rendtorff, Rolf. Das überlieferungsgeschichtliche Problem des Pentateuch. BZAW 147. Berlin: de Gruyter, 1977.

. Das Alte Testament: Eine Einführung. Neukirchen-Vluyn: Neukirchener Verlag, 1988.

Römer, Thomas. ,Zwischen Urkunden, Fragmenten und Ergänzungen: Zum Stand der Pentateuchforschung, “ $Z A W 125$ (2013): 2-23.

Schmid, Konrad. „Die Josephsgeschichte im Pentateuch.” Seiten 83-118 in Abschied vom Jahwisten: Die Komposition des Hexateuch in der jüngsten Diskussion. Hg. Jan C. Gertz, Konrad Schmid, und Markus Witte. BZAW 315. Berlin: de Gruyter, 2002.

. „Genesis in the Pentateuch.” Seiten 27-50 in The Book of Genesis:

Composition, Reception, and Interpretation. Hg. Craig A. Evans, Joel N. Lohr, und David L. Petersen. VTSup 152. Leiden: Brill, 2012.

Schmidt, Lothar. „Väterverheißungen und Pentateuchfrage.“ $Z A W 104$ (1992):1-27.

Seebass, Horst. Genesis II/1: Vätergeschichte I (11,27-22,24). Neukirchen-Vluyn:

Neukirchener Verlag, 1997.

. Genesis II/2: Vätergeschichte II $(23,1-36,43)$. Neukirchen-Vluyn:

Neukirchener Verlag, 1999.

. Genesis III: Josephsgeschichte (37,1-50,26). Neukirchen-Vluyn: Neukirchener Verlag, 2000.

Ska, Jean-Louis. Introduction à la lecture du Pentateuque. Clés pour l'interprétation des cinq premiers livres de la Bible. Brüssel: Éditions Lessius, 2000.

Steinberg, Naomi A. „The World of the Family in Genesis.” Seiten 279-300 in The Book of Genesis: Composition, Reception, and Interpretation. Hg. Craig A. Evans, Joel N. Lohr, und David L. Petersen. VTSup 152. Leiden: Brill, 2012. 
Tal, Abraham und Moshe Florentin. The Pentateuch: The Samaritan Version and the Masoretic Version. Tel Aviv: The Haim Rubin Tel Aviv University Press, 2010. Van Seters, John. Abraham in History and Tradition. New Haven: Yale University Press, 1975.

Warning, Wilfried. Literary Artistry in Leviticus. BibInt 35. Leiden: Brill. 1999. . „Terminologische Verknüpfungen und Genesis 15.” Hen 23 (2001): 3-9. . „Terminological Patterns and Genesis 17.” HUCA 70/71 (2001): 93-108. . „Terminological Patterns and Genesis 39.” JETS 44 (2001): 409-419. . „Terminological Patterns and Genesis 24.” EstBíb 60 (2002): 51-76. . "Terminological Patterns and the Book of Esther." OTE 15 (2002): 491496. . „Terminological Patterns and the Decalogue.” ZAW 118 (2006): 513-522.

Wenham, Gordon J. Genesis 1-15. WBC 1. Waco: Word Books, 1987. . Genesis 16-50. WBC 2. Waco: Word Books, 1994.

Witte, Markus. Die biblische Urgeschichte: Redaktions- und theologiegeschichtliche Beobachtungen zu Genesis 1,1-11,26. BZAW 265. Berlin: de Gruyter, 1998.

Whybray, R. Norman. Introduction to the Pentateuch. Grand Rapids: Eerdmans, 1995.

Zenger, Erich, u.a. Einleitung in das Alte Testament. 7. Aufl. Stuttgart: Kohlhammer, 2008.

Ziemer, Benjamin. Abram - Abraham: Kompositionsgeschichtliche Untersuchungen zu Genesis 14, 15 und 17. BZAW 350. Berlin: de Gruyter, 2005. . ,Die aktuelle Diskussion zur Redaktionsgeschichte des Pentateuch und die empirische Evidenz nach Qumran.“ $Z A W 125$ (2013): 383-399.

Dr Wilfried Warning, Rhönstraße 4, 64560 Riedstadt, Deutschland. Email: wiwa.vehlen@t-online.de. 